\title{
Real-time access to prescription drug monitoring databases
}

\author{
Barth Wilsey MD, Hannah Prasad BS
}

See related research article by Dormuth and colleagues on page E852 and at www.cmaj.ca/lookup/doi/10.1503/cmaj.120465

$\mathrm{T}$ he $C M A J$ article by Dormuth and colleagues advocates the implementation of centralized prescription monitoring to reduce inappropriate prescribing of benzodiazepines and opioids. ${ }^{1}$ According to the Canadian Centre on Substance Abuse, such surveillance is necessary to inform effective interventions for curtailing misuse and abuse. ${ }^{2}$

This issue is important to Canada as the second largest consumer of prescription opioids on a per capita basis, just behind the United States. ${ }^{2}$ Indeed, the US is dealing with a public health crisis of inadvertent overdoses with prescription drugs. ${ }^{3}$ As a result, there is unprecedented interest in centralized prescription networks or, as they are known in the US, prescription drug monitoring programs. The underlying rationale for these programs is to reduce rates of abuse and misuse, nonmedical use of controlled substances and prescription drug overdoses by curtailing doctor and pharmacy shopping by patients

The article by Dormuth and colleagues is important, because it adds to a growing body of literature supporting real-time access to data from prescription drug monitoring programs. In this regard, it is worthwhile to compare their study with that of Paulozzi and colleagues, ${ }^{4}$ who studied 19 state-level prescription drug monitoring programs. They concluded that, although such programs have the potential to be an important tool in preventing the nonmedical use of prescription drugs, their impact is not seen in drug overdose mortality; that the effect on the overall consumption of opioids is minimal; and the ways in which the data from such programs are used should be improved if there is to be an effect on overdoses from prescription drugs. ${ }^{4}$

Why this conclusion is at odds with that of Dormuth and colleagues can be understood by examining the reporting practices of centralized databases. Simply put, the studies differed in the degree of access health care providers had to the data from the monitoring programs. Many of the state-level programs studied by Paulozzi and colleagues did not allow or foster access by health care professionals. ${ }^{5}$ However, the program examined by Dormuth and colleagues allowed pharmacists real-time access to prescription data.

Forty-nine states currently have prescription drug monitoring programs or have passed legislation authorizing their formation. Not surprisingly, health care practitioners are being encouraged to use the data in these programs. ${ }^{6}$ However, clinicians do not use the databases to the greatest extent possible. As a result, the US Department of Health and Human Services is funding the "Enhancing Access to Prescription Drug Monitoring Programs" project. This undertaking stems from joint efforts between experts from both the public sector and private industry who participated in the White House Roundtable on Health Information Technology and Prescription Drug Abuse in June 2011. ${ }^{7}$ The project will fund pilot studies in Indiana and Ohio to determine whether health information technology can help increase the effectiveness of prescription drug monitoring programs by increasing providers' real-time access to the data. In the Indiana pilot, emergency department physicians will receive patients' histories concerning prescriptions for controlled substances from a centralized database. This aspect is important, because emergency departments are responsible for issuing almost $25 \%$ of prescriptions for controlled substances. ${ }^{7}$ In the Ohio pilot, drug risk indicators will be included in electronic health records, thus permitting the measurement of how this knowledge influences clinical decision-making.

Some of the evidence that provides the rationale for these experiments comes from a small

\section{KEY POINTS}

- Inadvertent overdoses from prescription drugs are a public health crisis

- Prescription drug monitoring programs will be an important part of preventing the misuse and abuse of controlled substances and fatal overdoses.

- Real-time access to the data in these programs is encouraged.

- A multifaceted approach by clinicians is needed to avoid penalizing patients who benefit from opioids or other controlled substances. 
observational study previously done in Ohio. In an effort to evaluate the potential negative impact of access to prescription drug monitoring programs on patient care in emergency departments, Baehren and colleagues ${ }^{8}$ reviewed clinical records associated with patients whose prescriptions were entered into Ohio's drug monitoring database. Real-time access to patient-specific records changed practitioners' prescribing practices for opioids in $41 \%$ of interactions. Accessing the database resulted in decreased or no opioid prescriptions after $61 \%$ of the queries, but an increase after the remaining $39 \%$ of queries. These results suggest that the targeted use of drug monitoring programs by prescribers does not result in an indiscriminate decrease in the administration of pain medications.

Doctor- and pharmacy-shopping behaviours have been defined using a variety of cut-offs for classifying a patient as having a potential to misuse or mismanage controlled substances that would warrant further evaluation. Published thresholds for such behaviours vary by number of providers or pharmacies seen by a single patient to obtain any opioid in a given period. However, because the number of providers or pharmacies are not direct measures of abuse or misuse, such information should be put into a clinical context. There may be justifiable reasons for patients to use multiple providers: they may have either changed clinicians, obtained medications from a practitioner covering for their customary provider or received appropriate treatment from multiple practitioners (e.g., a dentist and an emergency department physician). As with imaging and laboratory reports, clinical correlation by practitioners familiar with the patient's history is necessary to guide interactions with a patient who has used multiple health care providers or pharmacies.

In addition to information from prescription drug monitoring programs, there are several tools available to practitioners attempting to prescribe controlled substances judiciously. These include risk stratification, screening for aberrant behaviours and the use of highly structured approaches (e.g., opioid agreements, frequent visits, urine screening, pill or patch counts, psychoeducational sessions). ${ }^{9}$ Practitioners may also educate patients about not diverting drugs to friends or family, advocate storing drugs securely and use abuse-deterrent formulations, when feasible. ${ }^{8}$ National drug abuse authorities have opined that, in the effort to curtail abuse and misuse,

[w]e should not jeopardize or even restrict proper physician prescribing of opioids, nor should there be reduced accessibility to opioid medications for patients who need them. ${ }^{10}$

It is important for health care providers to strive to balance society's desire to curb drug abuse and misuse with the potential benefits of opioids and other controlled substances to individual patients.

\section{References}

1. Dormuth CR, Miller TA, Huang A, et al.; the Canadian Drug Safety and Effectiveness Research Network. Effect of a centralized prescription network on inappropriate prescriptions for opioid analgesics and benzodiazepines. CMAJ 2012;184:E852-56.

2. Buckley N, Davison C, Handford C, et al. Addressing prescription drug misuse in Canada: national dialogue summary report. Ottawa (ON): Canadian Centre on Substance Abuse; 2012. Available: www.ccsa.ca/Eng/Priorities/Prescription-Drug-Misuse/2012 -National-Dialogue-on-Prescription-Drug-Misuse/Pages/default .aspx (accessed 2012 Aug. 30).

3. Vital signs: overdoses of prescription opioid pain relievers United States, 1999-2008. Morb Mortal Wkly Rep 2011;60: 1487-92.

4. Paulozzi LJ, Kilbourne EM, Desai HA. Prescription drug monitoring programs and death rates from drug overdose. Pain Med 2011; 12:747-54.

5. Green TC, Zaller N, Rich J, et al. Revisiting Paulozzi et al.'s "Prescription drug monitoring programs and death rates from drug overdose." Pain Med 2011;12:982-5.

6. Katz N, Houle B, Fernandez KC, et al. Update on prescription monitoring in clinical practice: a survey study of prescription monitoring program administrators. Pain Med 2008;9:587-94.

7. New health IT effort aimed at reducing prescription drug abuse to be tested in Indiana and Ohio. Office of National Coordinator (ONC)/Substance Abuse and Mental Health Services Administration (SAMHSA); 2012. Available: www.hhs.gov/news/press /2012pres/06/20120621c.html (accessed 2012 Aug. 25).

8. Baehren DF, Marco CA, Droz DE, et al. A statewide prescription monitoring program affects emergency department prescribing behaviors. Ann Emerg Med 2010;56:19-23.

9. Executive summary: Tufts Health Care Institute Program on Opioid Risk Management. Summit meeting on prescription monitoring research update. Boston (MA): Tufts Health Care Institute; 2009. Available: www.thci.org/opioid/apr09docs/PMP $\% 20$ Executive\%20Summary.pdf (accessed 2012 Aug. 25).

10. Volkow ND, McLellan TA. Curtailing diversion and abuse of opioid analgesics without jeopardizing pain treatment. JAMA 2011;305:1346-7.

Affiliation: From the Department of Physical Medicine and Rehabilitation, University of California Davis Medical Center, Sacramento, Calif.

Contributors: Both of the authors made substantial contributions to the analysis and interpretation of the data for this commentary; they each revised the commentary critically for important intellectual content and provided final approval of the version submitted for publication. 